\title{
Discussion of Analyzing Speech to Detect Financial Misreporting
}

F E N G L I *

\section{Introduction}

One of the most important tasks facing investors, auditors, and regulators is to identify misreporting by managers, preferably using ex ante signals. Hobson, Mayew, and Venkatachalam [2012] (henceforth, HMV) examine the use of CEOs' voice markers of cognitive dissonance for detecting financial misreporting. Prior research on financial misreporting detection has focused mostly on financial measures, and, more recently, nonfinancial performance measures. HMV innovate by bringing a new perspective into this field. They motivate this research question with the findings in the psychology literature that nonverbal vocal markers can help discriminate deceivers from truth tellers. They focus on a specific type of vocal marker in the paper-cognitive dissonance-based on prior evidence (e.g., Mazar, Amir, and Ariely [2008]) that misreporting leads to cognitive dissonance, which is the feeling of psychological discomfort when one's actions and beliefs are discrepant.

To measure managers' cognitive dissonance, HMV rely on a proprietary software package, Layered Voice Analysis (LVA). The first step in the empirical tests is to validate the measure as capturing cognitive dissonance. They

\footnotetext{
*University of Michigan. I would like to thank Jason V. Chen, Merle Erickson (the Editor), Yu Huang, Nemit Shroff, and the participants of the Journal of Accounting Research 2011 Annual Conference for their comments. I gratefully acknowledge the Harry Jones Endowment at the University of Michigan for financial support.
} 
utilize an experimental setting in which they induce misreporting behavior by lab subjects. They then calibrate the amount of cognitive dissonance for the subjects and examine whether it is correlated with the cognitive dissonance measure based on LVA. The results indicate that the LVA indicators are indeed positively associated with cognitive dissonance. In the second step, HMV use archival data and find that the cognitive dissonance exhibited by CEOs during conference calls positively predicts future irregular restatements.

HMV address an important research question with an innovative approach. Although there are numerous studies in the accounting literature that predict financial misreporting, they mostly focus on financial data (Dechow et al. [2011]), nonfinancial performance data (Brazel, Jones, and Zimbelman [2009]), or verbal cues (Larcker and Zakolyukina [2012]). Anecdotal evidence, however, suggests that practitioners (e.g., the CIA) have been using nonverbal cues to detect financial fraud, suggesting that nonverbal cues could be useful in such a setting. HMV are the first to examine nonverbal cues from conference calls to detect financial misreporting. The use of both lab experiments and archival data in the empirical tests is also unique.

In this discussion, I focus on three aspects. First, I discuss some possible alternative explanations of the findings in HMV. In particular, it is possible that the cognitive dissonance metric in the paper captures other psychological features, rather than cognitive dissonance per se. Next, I discuss sample selection issues with the empirical tests and their implications. Lastly, I discuss possible future research opportunities.

\section{Alternative Explanations}

Due to data limitation and other constraints, HMV cannot completely rule out alternative explanations based on their research design. In particular, the biggest challenge to interpreting the results is what the LVA marker captures conceptually and whether it indeed measures cognitive dissonance. From a pure practical and utilitarian perspective (e.g., helping analysts and investors detect possible financial misreporting), this is not a serious concern. However, from a theoretical perspective, this distinction is an important one since it influences how we understand and interpret the evidence. Moreover, it will affect how the literature proceeds. I discuss some alternative interpretations of the empirical results in this section.

\subsection{FEAR}

HMV correctly point out that a necessary condition for them to observe valid evidence consistent with their hypothesis is that the vocal dissonance markers based on the LVA software must capture cognitive dissonance stemming from misreporting without significant measurement error. The lab experiments in HMV are designed to validate this assumption. 


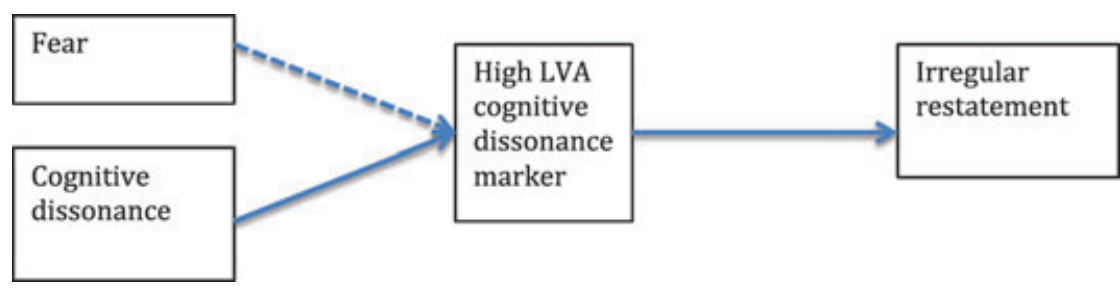

FIG. 1.-The links between fear, cognitive dissonance, and misreporting in conference call setting.

The cognitive dissonance theory, developed by Festinger [1957], is about the relationships among the different cognitions human beings have. The cognitions may be the knowledge about an attitude, an emotion, a behavior, a value, etc. When people hold a multitude of cognitions simultaneously, these cognitions could be dissonant or inconsistent with one another. Cognitive dissonance is the tense feeling that occurs when a person holds two equally valid but conflicting views (Festinger [1957], Aronson [1997]).

Misreporting could lead to cognitive dissonance because the behavior of misreporting is inconsistent with people's belief that they are honest. However, it is possible that other psychological characteristics and cognitive dissonance might be codetermined and these other characteristics could potentially explain the empirical results. There are many other different types of nonverbal cues that might relate to misreporting, such as the fear of being detected and the consequences associated with being detected. In other words, when a person misreports, she is also likely to be fearful of the potential consequences that may arise from being caught.

Aware of the confounding fear factor, HMV design their experiment in such a way that the experiment subjects retain their test sheets; as a result, they do not feel fearful of being detected. By doing so, HMV can attribute the observed cognitive dissonance based on the LVA software to the cognitive dissonance induced by the research design.

However, this design does not rule out the possibility that the LVA cognitive dissonance marker also captures the feeling of fear or other psychological traits (i.e., "A causes B" does not mean that "B cannot be caused by C.") The lab experiments in HMV demonstrate that cognitive dissonance leads to a high LVA dissonance marker, which by design cannot be due to the subjects' fear of being caught. However, as illustrated in figure 1, in a conference call setting, a high LVA cognitive dissonance marker may be caused either by the CEOs' cognitive dissonance or by their fear of being detected. The key difference between the HMV lab setting and the conference call setting is that, in the conference call settings, the CEOs are likely to be fearful (in addition to potentially having cognitive dissonance).

To summarize, the experiments in HMV nicely show that the LVA marker indeed captures cognitive dissonance, but they do not show whether (or how much) it also captures other psychological characteristics such as fear of detection. Given the proprietary nature of the LVA software, it is difficult 
for researchers to assess whether it captures cognitive dissonance only. The possibility that CEOs' fear of being caught and other psychological traits potentially captured by the LVA dissonance marker may explain the archival findings documented by HMV is an important alternative explanation. This is especially important given that fear of being caught and deterrence are the central focus in the economic theory of crime (Tullock [1974], Levitt [1998]).

\subsection{UNCERTAINTY}

Cognitive dissonance is likely to be more severe when human beings face more uncertainty in their decision-making process (Lévy-Garboua and Blondel [2002]). Uncertainty can relate to cognitive dissonance in two ways. First, as HMV point out, uncertainty can cause managers to have cognitive dissonance. This is because, in a more uncertain environment, a firm's performance is less predictable and the performance may not be consonant with the beliefs held by the manger (e.g., the belief that hard work leads to better performance). Second, the observed high LVA cognitive dissonance score may capture a CEO's other responses to uncertainty (e.g., anxiety or revision of beliefs), rather than her cognitive dissonance.

Note that HMV are aware of the first issue. Because their research question is about whether misreporting-related cognitive dissonance is useful for detecting misreporting, HMV control for uncertain environments using firm size and stock return volatility to isolate the effect of the component of the CEO's cognitive dissonance that is due to misreporting.

However, the second issue, that the LVA measure could capture managers' other responses to uncertainty, rather than their cognitive dissonance, could lead to another possible alternative explanation of the results. Specifically, the results in HMV are also consistent with the following: (1) firms with more business or information uncertainty are more likely to commit financial fraud and get caught, and (2) CEOs of such firms face more scrutiny in conference calls and, when this happens, the CEOs are reminded of the uncertainty and the LVA marker picks up their response to such uncertainty.

This alternative interpretation argues that the link between misreporting and the LVA marker is not driven by the cognitive dissonance due to misreporting. Rather, the positive association between misreporting and the LVA measure are both determined by uncertainty. Under this interpretation, it is not enough to control for observable uncertainty measures (such as return volatility) in the empirical tests because the LVA dissonance marker could be a better measure of CEOs' reaction to uncertainty than the uncertainty variables.

\section{Selection Issues}

I discuss two sample selection issues with the empirical tests. It is important to note that these two selection issues are not caveats for HMV alone; rather, they are common issues in the literature. 
The first sample selection issue is related to the subjects in the lab experiment. To examine the construct validity of the LVA marker as a measure for cognitive dissonance in a conference call setting, the ideal experiment should mimic the conference call environment. Concerns about selection arise here because the subjects in the HMV experiment, who were students from two large public universities and voluntarily signed up for the experiments, may not represent the CEOs in terms of psychological behavior. Therefore, the validation conclusion based on the lab experiments may not be generalizable to CEOs in the conference call settings. Of course, this external validity concern applies to most lab experiments in the accounting literature, but it may be more significant in the HMV setting. This is because, compared to the average population, CEOs are more successful; they are perhaps more overconfident, and have better speech training and therefore are less subject to cognitive dissonance.

If the LVA dissonance marker does capture CEO cognitive dissonance in the conference calls, then this sample selection issue is not a big concern because it biases against finding significant results in the archival tests. In other words, if the CEOs have relatively little cognitive bias, this implies smaller variation in the observed cognitive dissonance and lower power for detecting misreporting. However, the bigger concern is that, if the LVA marker also captures other characteristics of the speaker (e.g., fear as discussed in the previous section), then it may capture cognitive dissonance for the students in the laboratory setting but something else for the CEOs because of the fundamental differences between the two groups of people.

The second selection issue relates to the archival tests. Specifically, HMV gather financial misreporting from the Audit Analytics restatements database and differentiate intentional (i.e., irregularities) from unintentional (i.e., errors) restatements. In the empirical tests, HMV focus on the irregular restatements. The selection issue arises because the firms that were caught for misreporting (i.e., the restatement firms) may not represent the true population of firms that misreport. Specifically, the firms that were caught for misreporting may have managers who are bad at lying; therefore, such CEOs tend to have higher cognitive dissonance scores in conference calls.

An implication of this selection bias is that cognitive dissonance may be useful for detecting the "bad cheaters," but may be of limited power for catching the "good cheaters" who don't show up in the restatement sample. The importance of this sample selection issue is a function of the research objective. If the objective is to quickly detect financial misreporting (e.g., before the SEC catches the misreporting), then this sample selection issue does not matter because the LVA software can potentially catch the misreporters that would most likely be caught in the future in a more timely fashion. If, on the other hand, the research objective is to detect all the misreporters (regardless of whether they are caught and have to restate the numbers), then the selection bias in the sample is a caveat of the study 
because the empirical results do not speak about the entire misreporting sample.

\section{Future Research}

HMV provides very interesting evidence suggesting that cognitive dissonance markers could be used to detect financial misreporting. This opens up many possibilities for future research. ${ }^{1}$

\subsection{EXTENSION OF HMV}

A nice and distinctive feature of HMV is that it combines the validity check using a lab setting and the archival empirical study based on the validated measure. Future research could extend HMV in both the experimental part and the archival tests part.

One direction for future research could be to more carefully examine what drives the LVA cognitive dissonance marker in a lab setting to further examine its construct validity. As discussed above, by design the experiments in HMV cannot test whether (or how much of) the LVA cognitive dissonance marker can be driven by the fear of detection. It might be interesting to intentionally introduce fear and variation in other psychological traits (such as overconfidence) in the experiments to examine how much of the variation in the LVA dissonance marker is due to these other factors.

Another possible direction is to examine the interaction of the nonverbal cues, as examined by HMV, and the verbal cues and how they complement each other and help jointly detect financial misreporting. Current research examines the financial statement information, verbal cues, and nonverbal cues separately to detect financial fraud. HMV find that the cognitive dissonance markers play an incremental role to the verbal cues and financial statement data. Therefore, a model that includes all three pieces of information is likely to have increased power for empirical tests.

In addition, because of the limited availability of the conference call audio files, it is challenging to conduct archival empirical tests exploring cognitive dissonance using such data. It is therefore interesting to explore whether verbal cues can also be used to capture managerial cognitive dissonance.

Further, future research can extend HMV by utilizing the fraud triangle framework (Turner, Mock, and Srivastava [2003]). The fraud triangle theory argues that three conditions are generally present when fraud occurs: Incentive/Pressure, Opportunity, and Attitude/Rationalizations. The cognitive dissonance studied in HMV is closely related to the "Attitude/Rationalizations" component. Combining the dissonance marker with the variations in incentives and opportunities to detect financial misreporting could be fruitful for future research.

\footnotetext{
${ }^{1}$ One technical difficulty for follow-up studies is that the audio files of conference calls are not easily available to researchers.
} 


\subsection{NOVELTY-BASED DETECTION}

Human beings adapt to their environment. If a CEO knows that her verbal or nonverbal cues may reveal her misreporting behavior, she can intentionally change her behavior through training. In addition, as discussed in the previous section, "good cheaters" may be good at controlling cognitive dissonance and therefore traditional techniques used to detect cognitive dissonance may have low power for such people.

A possible direction for future research is then to build on the novelty detection concept in psychiatry and explore the verbal and nonverbal cues of market participants in settings where novel information is presented to these participants to detect misreporting. Novel information represents a deviation from the expected likelihood of an event on the basis of both previous information and internal estimates of conditional probabilities. Psychiatry research has documented that human brains respond to novel information and this response can happen without the subjects' awareness (Berns, Cohen, and Mintun [1997]).

The novelty detection literature suggests that researchers should look for "surprises" in conference call or other settings to search for cognitive dissonance markers. In other words, focusing on the cognitive dissonance caused by novel information instead of the average cognitive dissonance throughout the conference call may increase the detection power. For instance, when analysts ask a CEO an unexpected question or a question related to earnings quality, the CEO's cognitive dissonance induced by misreporting may be substantially higher (even if she is not aware of it). Therefore, by focusing on the novel information researchers may be able to better measure and exploit the cognitive dissonance to detect misreporting.

\subsection{ECONOMIC CONSEQUENCES OF COGNITIVE DISSONANCE}

Future research might also study the economic consequences of cognitive dissonance more systematically. Akerlof and Dickens [1982] modify the traditional rational decision-making model and construct a decision model by incorporating cognitive dissonance. They show that several seemingly puzzling economic phenomena can be explained when a person's cognitive dissonance is taken into consideration.

The evidence in HMV suggests that the LVA marker is a valid measure of cognitive dissonance. This measure can be used to study the economic consequences of managerial cognitive dissonance. While the evidence in HMV suggests that CEOs who misreport have cognitive dissonance, the next step is to examine whether the cognitive dissonance has implications for managerial decision making. For instance, when a CEO makes acquisitions using her firm's overvalued equity, she may experience some sort of cognitive dissonance. The implications of this cognitive dissonance for the postacquisition performance are interesting to examine. 


\section{Conclusion}

HMV show that CEOs' cognitive dissonance can be used to predict financial misreporting. Their study is innovative and addresses an important question. The evidence in HMV both makes a theoretical contribution and has practical implications. From a theoretical perspective, HMV demonstrate that nonverbal cues can have incremental predictive power to detect financial misreporting and that incorporating managerial cognitive dissonance could be useful in future theoretical modeling of corporate decision making. From a practical perspective, HMV demonstrate that utilizing the nonverbal cues in conference calls may provide additional information for investors, auditors, and regulators to detect financial misreporting.

Future research is needed to further validate the LVA software-based cognitive dissonance marker. The use of both experimental design and archival data to test economic hypotheses may also be a fruitful model for future research.

\section{REFERENCES}

Akerlof, G. A., AND W. T. DickEns. "The Economic Consequences of Cognitive Dissonance." American Economic Review 72 (1982): 302-19.

Aronson, E. "The theory of Cognitive Dissonance: The Evolution and Vicissitudes of an Idea," in The Message of Social Psychology, edited by C. McGarty and S. A. Haslam. Oxford: Blackwell Publishers, 1997: 20-36.

Berns, G. S.; J. D. Cohen; And M. A. Mintun. "Brain Regions Responsive to Novelty in the Absence of Awareness." Science 276 (1997): 1272-75.

BRAZEL, J.; K. Jones; And M. Zimbelman. "Using Nonfinancial Measures to Assess Fraud Risk." Journal of Accounting Research 47(2009): 1135-66.

Dechow, P. M.; W. GE; C. R. LARSOn; AND R. G. SloAn. "Predicting Material Accounting Misstatements." Contemporary Accounting Research 28 (2011): 17-82.

Festinger, L. 1957. A Theory of Cognitive Dissonance. Stanford, CA: Stanford University Press.

Hobson, J. L.; W. J. MayeW; And M. Venkatachalam. "Analyzing Speech to Detect Financial Misreporting." Journal of Accounting Research 50 (2012): 349-392.

LARCKER, D., AND A. ZAKOlYUKINA. "Detecting Deceptive Discussion in Conference Calls." Journal of Accounting Research 50 (2012): 495-540.

LevitT, S. "Juvenile Crime and Punishment." Journal of Political Economy, 106 (1998): 1156-85.

LÉVy-GarbouA, L., AND S. BlOndEL. "On the Rationality of Cognitive Dissonance." The Expansion of Economics: Towards an Inclusive Social Science, edited by S. Grossbard-Schechtman and C. Clague. Armonk, NY: MESharpe, Inc., 2002.

MAZAR, N.; O. AMIR; AND D. ARIELY. "The Dishonesty of Honest People: A Theory of SelfConcept Maintenance.” Journal of Marketing Research 45 (2008): 633-44.

Tullock, G. "Does Punishment Deter Crime?" Public Interest, 36 (1974): 103-11.

Turner, J. L.; T. J. Mock; AND R. P. SRIVASTAVA. "An Analysis of the Fraud Triangle." AAA Round Table Paper, 2003. Available at http://aaahq.org/audit/midyear/03midyear/ papers/Research\%20Roundtable\%203-Turner-Mock-Srivastava.pdf. 